\title{
Whirl Flutter Stability and Its Influence on the Design of the Distributed Electric Propeller Aircraft X-57
}

\author{
Christian B. Hoover* and Jinwei Shen ${ }^{\dagger}$ \\ Andrew R. Kreshock \\ University of Alabama, Tuscaloosa, AL \\ Army Research Laboratory, Hampton, VA \\ Bret K. Stanford ${ }^{\S}$ and David J. Piatak ${ }^{\S}$ and Jennifer Heeg ${ }^{\llbracket}$ \\ NASA Langley Research Center, Hampton, VA
}

\begin{abstract}
This paper studies the whirl flutter stability of the NASA experimental electric propulsion aircraft designated the X-57 Maxwell. whirl flutter stability is studied at two flight conditions: sea level at 2700 RPM to represent take-off and landing and 8000 feet at 2250 RPM to represent cruise. Two multibody dynamics analyses are used: CAMRAD II and Dymore. The CAMRAD II model is a semi-span X-57 model with a modal representation for the wing/pylon system. The Dymore model is a semi-span wing with a propeller composed of beam elements for the wing/pylon system that airloads can be applied to. The two multibody dynamics analyses were verified by comparing structural properties between each other and the NASTRAN analysis. For whirl flutter, three design revisions of the wing and pylon mount system are studied. The predicted frequencies and damping ratio of the wing modes show good agreements between the two analyses. Dymore tended to predict a slightly lower damping ratio as velocity increased for all three dynamic modes presented. Whirl flutter for the semi-span model was not present up to 500 knots for the latest design, well above the operating range of the X-57.
\end{abstract}

\section{Introduction}

This paper studies the whirl flutter stability of the NASA experimental electric propulsion aircraft, designated as the X-57 Maxwell. This aircraft is designed to be quieter, lighter, more efficient and environmentally friendly than current turboprops. ${ }^{1}$ While currently limited to low-power missions, the development of electric aircraft is seen as a crucial step towards future green aircraft with wide applications. The X-57 is the latest iteration of the Scalable Convergent Electric Propulsion Technology and Operations Research (SCEPTOR) vehicle and is based on the Tecnam P2006T aircraft. Compared to the two-propeller Tecnam P2006T, the X-57 has reduced wing area along with an additional twelve wing-integrated propellers. The objective behind 14 propellers is that in low speed flight, such as take-off and landing, the down-wash of the smaller 12 inboard propellers would help the wing generate lift in what would otherwise be stall conditions. Under high speed flights, such as in cruise, the X-57 will be operating with only the two larger outboard propellers active.

As with any new aircraft, it is imperative that any aeroelastic instabilities do not occur inside the flight envelope. A particular concern for the X-57 is the potential for whirl flutter. This aeroelastic instability is caused by the propeller aerodynamics, which drives the airframe/pylon motions to become unstable. Whirl flutter, although analytically discovered by Taylor and Browne in $1938,{ }^{2}$ became a major concern in the 1960s following the two fatal accidents of the Electra aircraft. ${ }^{3}$ The Electra failures were caused by a structural failure in a pylon mount that weakened its stiffness and ultimately led to the onset of whirl flutter. The Electra accidents caused a newfound interest in whirl flutter which was subsequently studied at the NASA Langley Research Center. ${ }^{4}$ A full review of whirl flutter was conducted by William Reed III at NASA Langley, where theoretical and experimental results were correlated. ${ }^{5}$ While a reduction in pylon stiffness caused the fatal Electra accidents, the X-57 is designed with propellers mounted on thin, highly efficient and low weight wings which may potentially present the conditions prone to whirl flutter.

\footnotetext{
*Graduate Student, Department of Aerospace Engineering and Mechanics. Student Member AIAA.

${ }^{\dagger}$ Assistant Professor, Department of Aerospace Engineering and Mechanics. Senior Member AIAA.

₹Aerospace Engineer, Vehicle Technology Directorate. Member AIAA.

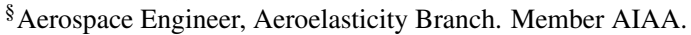

IIAerospace Engineer, Aeroelasticity Branch. Associate Fellow AIAA.
} 
This paper presents the whirl flutter stability study of the NASA X-57 Maxwell through two multibody dynamics analyses: CAMRAD II ${ }^{6}$ and Dymore. ${ }^{7}$ Both CAMRAD II and Dymore have demonstrated the capability of predicting tiltrotor whirl flutter. ${ }^{8-10}$ CAMRAD II, along with another multibody dynamics, RCAS, was shown to predict the whirl flutter boundary of a tiltrotor. ${ }^{11}$ CAMRAD II and Dymore showed good comparison in the study of a UAV tiltrotor in helicopter, airplane, and transition modes, as well as maneuvering. ${ }^{12}$ CAMRAD II, Dymore, and RCAS were also shown to capture the whirl flutter trends of a tiltrotor in cruise and the proprotor loads at various pylon conversion angles. ${ }^{13}$

The objectives of this study are to develop and verify the multibody dynamics models of the X-57 and carry out a whirl flutter study of several design revisions for this experimental aircraft. The model development is through a modular approach where the fixed system and rotating system are developed and verified separately and then integrated to complete the semi-span model of the X-57. Isolated propeller loads, natural frequencies, and mode shapes of the wing/pylon system are used in the verification. Whirl flutter stability predictions are compared between the analyses to verify that they produce consistent results.

\section{Analytical Models}

The multibody dynamics codes used here, CAMRAD II and Dymore, have been widely used in the rotorcraft community, and applied in predicting tiltrotor whirl flutter. ${ }^{8-10}$ In order to test the robustness of the methodology and their abilities to model the whirl flutter, their predictions are correlated with each other. The conceptual drawing and key variables of the tip propeller for the X-57 Maxwell aircraft are shown in Figure 1. For investigation of whirl flutter stability, a semi-span structural model is developed to calculate the frequency and damping ratio of the wing symmetric modes.

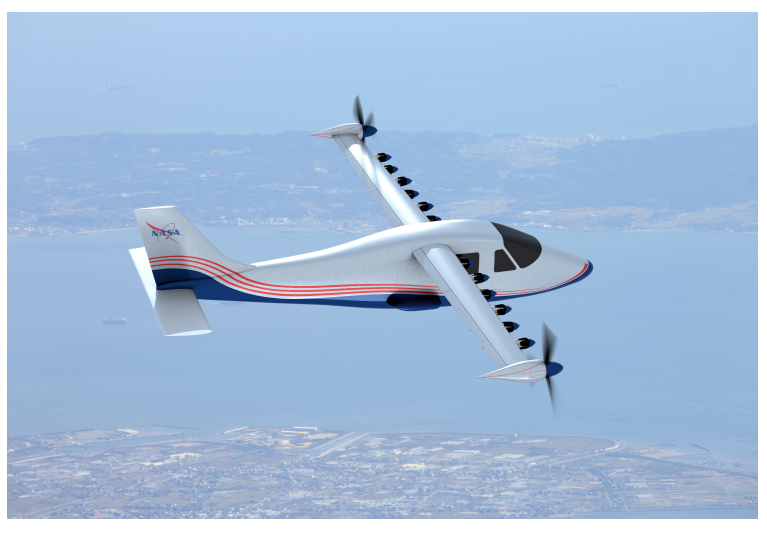

(a) Concept of X-57 Maxwell

\begin{tabular}{lc}
\hline \hline Property & Value \\
\hline Tip Prop Number of Blades & 3 \\
Tip Prop Diameter & $5 \mathrm{ft}$ \\
Take-Off RPM & 2700 \\
Cruise RPM & 2250 \\
Cruise Speed & $150 \mathrm{KTS}$ \\
Wing Semi-Span & $14 \mathrm{ft}$ \\
Wing Norminal Chord & $2.1 \mathrm{ft}$ \\
\hline \hline
\end{tabular}

(b) Key Aircraft Parameters

Figure 1: NASA X-57 Maxwell

\section{II.A. Analytical Models of X-57 Maxwell}

Dymore is a finite element (FE) based multibody dynamics code for the comprehensive modeling of nonlinear flexible multibody systems. ${ }^{14}$ The equations of equilibrium are derived in a Cartesian inertial reference, and constraints are modeled using the Lagrange multiplier technique. This leads to a system of differential-algebraic equations which is then solved using a robust time integration scheme. Dymore's element library includes rigid and deformable bodies as well as joint elements. Deformable bodies are modeled with the FE method, and the formulations of beams and shells are geometrically exact, i.e., they account for arbitrarily large displacements and finite rotations but are limited to small strains. The aerodynamic forces can be computed with the built-in lifting line theory or through coupling with an external aerodynamics code.

The Dymore model of the X-57 Maxwell aircraft, shown in Figure 2 includes the wing, pylon, and the tip propeller. The wing and propeller blades are modeled as elastic beams. The inboard propellers and the pylon and nacelle of the large tip propellers are modeled as rigid bodies with their appropriate inertial properties. The blade pitch bearing is modeled as a revolute joint. The pylon mount flexibility in yaw and pitch is captured with linear springs connecting the pylon to the wing. The aerodynamic forces acting on the propeller and wing are modeled 
with both quasi-steady and unsteady lifting line theories. The unsteady aerodynamics used in Dymore are based on the unsteady aerodynamics theory developed by Peters. ${ }^{15}$ A table lookup format is used to define the propeller aerodynamic coefficients. Rotor inflow is calculated with a three-dimensional nonlinear dynamic inflow model using three inflow states. The aerodynamic interaction between propeller and wing is ignored.

CAMRAD II is an aeromechanical analysis of helicopters and rotorcraft that incorporates several tools, including multibody dynamics, nonlinear FE, structural dynamics, and rotorcraft aerodynamics. ${ }^{6}$ The CAMRAD II X-57 Maxwell model includes a rigid propeller model and modal representations of the wing/pylon fixed systems. Propeller aerodynamics are modeled with the lifting line theory coupled with a linear inflow model. ${ }^{10}$

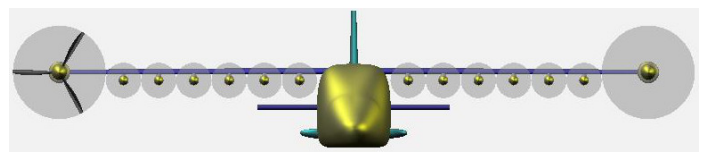

(a) X-57 Maxwell Illustration

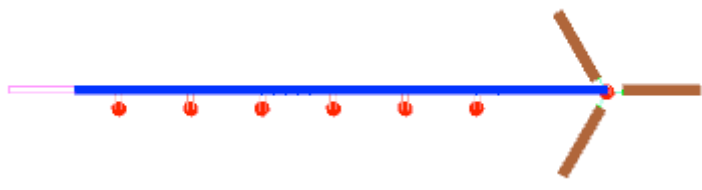

(b) Dymore X-57 Maxwell Semi-Span Model

Figure 2: Dymore X-57 Maxwell Semi-Span Model for Whirl Flutter Study

\section{II.B. Three Wing/Pylon Design Versions}

This study covers three versions of the wing/pylon system, and the effects of various design decisions on whirl flutter is studied. The three versions will be called in this paper Versions 1-3.

\section{II.B.1. Version 1}

Version 1 occurred during the initial wing design of the X-57 vehicle. The wing design and aeroelastic analyses were performed in concert, so that one would impact the other and presumably keep the design in a region of the design space with robust aeroelastic safety margins. This process necessitates that the structural model be analyzed at various levels of completion. The initial FE structural model of the X-57 wing included only primary components of the wing; the cruise propulsion system components (tip nacelle, pylon, propeller and motor) were represented solely by their mass properties. This included a full 3D FE model of the wing. For whirl flutter analysis in Dymore, an equivalent beam model was derived.

\section{II.B.2. Version 2}

Between Version 1 and Version 2, two substantial changes were made in the structural design and model. The close spacing observed for the first two modal frequencies in the Version 1 design was an aeroelastic concern, more from a wing flutter perspective than from a whirl flutter perspective. This led to incorporation of unidirectional fibers in the spar caps.

The second important development was that the design of the tip nacelle geometry, structure and propulsion system had advanced so that details could be included in the analytical model. The design of the tip nacelle included a firewall that served as a faceplate for mounting the motor for the tip propeller. This faceplate provides much of the structural stiffness between the propeller system and the nacelle, strongly influencing the in-plane behavior. Due to this being a local phenomenon the equivalent beam model required by the Dymore analysis relied on the flexibility of the tip pitch and yaw springs to partially account for this local deformation. However, because CAMRAD II uses a modal representation, it was able to include these effects.

\section{II.B.3. Version 3}

The firewall was stiffened in Version 3, substantially reducing the faceplate deformations. Additional design and modeling developments were incorporated, including more detailed representations of the control surfaces, control systems and cabling. The mode shapes and frequencies for Version 3 were generated using a FE model where the control surfaces and systems were removed, as was also done in Version 2. This was necessitated by the equivalent beam model process. The mass and inertial properties of the control surfaces were not incorporated into the beam model for Version 3. 


\section{II.C. Equivalent Beam Model}

Structural models of the wing configurations studied here consisted of NASTRAN FE models, ${ }^{16}$ with quad-dominant shell elements used to model the spar-rib wingbox. The wind-off structural mode shapes and natural frequencies could then be computed in NASTRAN. CAMRAD II is able to take this eigen-data as a direct input, to facilitate its whirl flutter analysis. In order to analyze the configuration in Dymore, however, equivalent beam models had to be created for each wingbox. Equivalent beam models were first constructed and verified in NASTRAN, and then re-constructed and re-verified in Dymore. The following steps were undertaken to construct each equivalent beam model:

1. The elastic axis of the wingbox is located, by dividing the wingbox into several spanwise sections. The root of each section is clamped, and vertical loads are applied to the tip, in order to find the force application location which produces a pure bending response, without torsion. This process is repeated until the elastic axis is mapped from wing root to wing tip.

2. Beam elements are laid-out along this elastic axis, and these beam elements are connected to the wingbox with mass-less interpolation elements.

3. Forces and moments are applied to each beam node. Because the beam grids are "spidered" to the wingbox (and because the beams do not yet have any stiffness properties), these forces are counteracted by the wingbox alone. As the wingbox deforms, the internal beam grids passively deform as well. Examining the relative deformation and rotation of adjacent beam nodes produces estimates for the bending and torsional stiffness of each beam element.

4. Mass properties of each beam element are computed from the known inertial properties of the wingbox. Concentrated masses are created for each nacelle location, and connected to the beam elements with rigid linkages.

5. The final beam model is "disconnected" from the wingbox, and the natural frequencies and mode shapes can be computed for the equivalent beam, for comparison with the eigen-data of the full wingbox model.

6. In order to further tune the modal response of the equivalent beam model to that of the wingbox model, an optimization process is conducted. The elastic and inertial properties of each beam element, as well as the pitch and yaw stiffness for two torsional springs at the wing tip (connecting the wing to the tip propeller) are optimized, in order to minimize a weighted objective function. This objective includes the sum of the differences in the two sets of natural frequencies (beam model and shell wingbox model), as well as the norm-difference between the modal assurance criterion (MAC) matrix and the identity matrix. If the two sets of mode shapes are identical, then the MAC matrix will become the identity matrix. This optimization is conducted with a genetic algorithm.

The development of the beam models for Versions 2 and 3 utilize this complete 6-step procedure. The beam model for Version 1 only uses steps 1-5, however, neglecting the tuning optimization.

\section{Analytical Results}

The analytical results include the verification of the X-57 Maxwell analytical models and the whirl flutter stability predictions. The important sub-components of the semi-span X-57 Maxwell analytical model, the fixed subsystem (wing/pylon) and the rotating subsystem (isolated propeller), are developed and verified separately by correlating the predictions among different analyses (Dymore, CAMRAD II, NASTRAN).

\section{III.A. X-57 Maxwell Analytical Model Verification}

The analytical model development is carried out component by component, through a modular approach with correlation among the analytical models at each step. Figure 3 illustrates the modular procedure applied to the semi-span X-57 Maxwell model. The comparison of predictions of characteristics of these key components among CAMRAD II, Dymore, and NASTRAN are presented below. NASTRAN analysis is used to validate the structural model of the wing/pylon system. The aerodynamic model of the propeller is correlated between CAMRAD II and Dymore. 


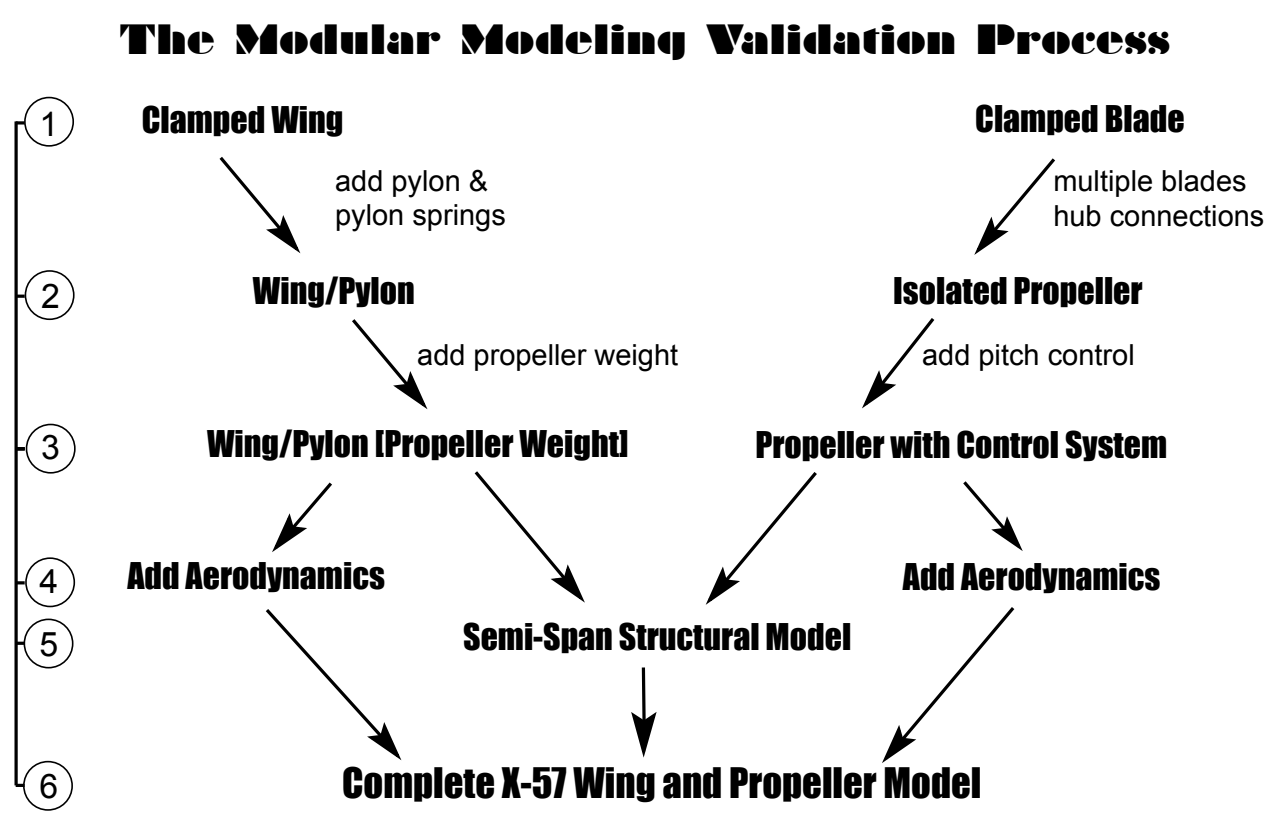

Figure 3: Modular Model Development Approach

\section{III.B. Equivalent Beam Model of Three Fixed System Versions}

This section compares the modal vibration results of the full FE wingbox model and the derived equivalent beam model.

\section{III.B.1. Version 1}

The mode shapes and frequencies for this FE model are shown in Figure 4. The first two mode shapes can be described qualitatively as wing vertical first bending mode $(1.8 \mathrm{~Hz})$ and wing inplane first bending mode $(2.8 \mathrm{~Hz})$. As noted above, these first two frequencies are relatively close to each other. Due to concerns about aeroelastic wing flutter problems, design changes were made in the next version to separate theses frequencies. It can also be seen in Figure 4 that the equivalent beam model is unable to correctly predict the torsional vibration shape of the sixth mode. This is due to the fact that Step 6 in Section II.C (additional tuning of the beam model) was not performed for this version.

\section{III.B.2. Version 2}

The unidirectional fibers in the spar caps raised the modal frequency of the first in-plane mode to $6.8 \mathrm{~Hz}$, as seen in Figure 5. It can also be seen that all six modes in Figure 5 agree well, both in terms of shape and frequency, between the equivalent beam and the full FE model, due to the tuning process not used in Version 1. Modal analysis of the firewall also revealed significant local deformation of the faceplate, particularly in the second in-plane bending mode, as shown in Figure 6. The tuned tip yaw spring used in the equivalent beam model is able to partially account for this relative deformation as well.

\section{III.B.3. Version 3}

Figure 7 shows the mode shapes and frequencies for Version 3, which were generated using a FE model where the control surfaces and systems were removed. This was necessitated by the equivalent beam model process. It can also be seen that the local deformation of the firewall faceplate seen in Figure 5 is less prevalent here.

\section{III.B.4. Wing/Pylon System}

NASTRAN FEM analysis is used for deriving the wing/pylon beam model used in the flexible multibody dynamic analyses for the whirl flutter study shown in Figure 8. The properties of the equivalent beam model are derived from the detailed X-57 Maxwell FEM model through a virtually experimental technique. This beam model was then 

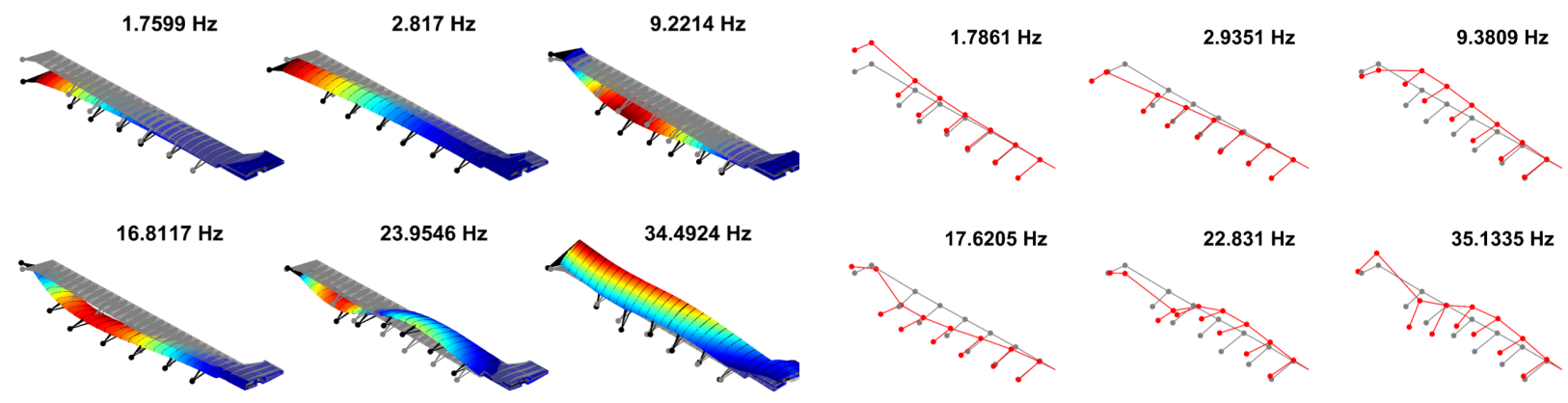

(a) Full FEM

(b) Beam Model

Figure 4: Version 1: Full FEM and Equivalent Beam Model
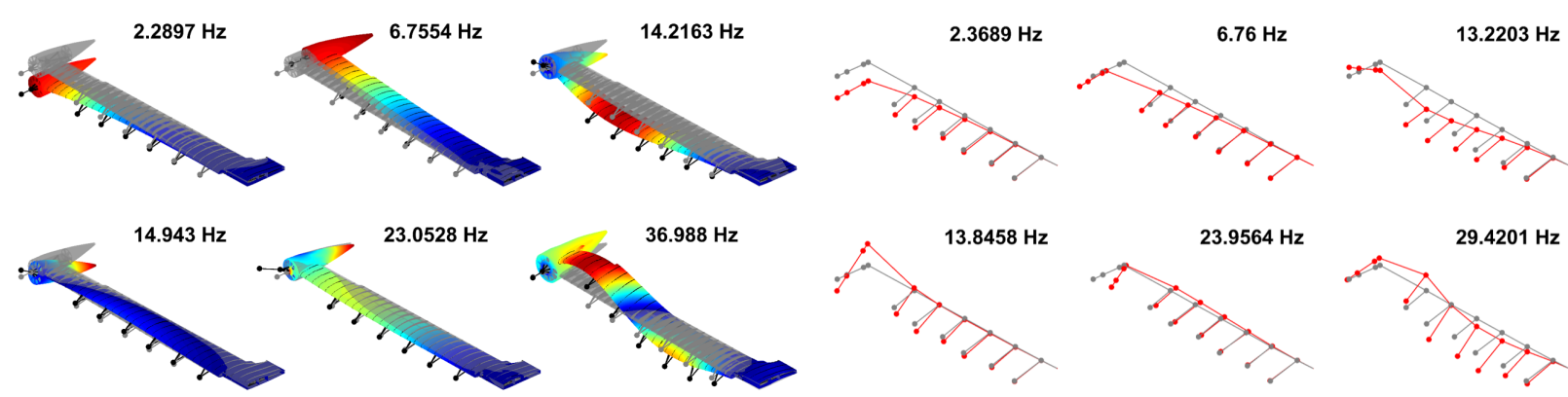

(a) Full FEM

(b) Beam Model

Figure 5: Version 2: Full FEM and Equivalent Beam Model

re-created in Dymore, and the predicted natural frequencies were compared to the NASTRAN calculations for the Version 3 configuration. Table 1 lists the first ten natural frequencies, and the maximum error between Dymore and the NASTRAN beam model predictions is less than 2.0\%. A graphical comparison of the frequencies is shown in Figure 9. Figure 10 compares the mode shapes of the first four modes of the wing/pylon model between the NASTRAN and Dymore predictions. These four modes are respectively: the first wing bending, inplane bending (knife-edge), second bending (out-of-plane), and torsion modes. These modes play critical roles in the dynamics of propeller whirl flutter stability, and the good agreements between the analytical predictions of the frequencies and mode shapes ensure accurate structural representation of the wing/pylon system.

\section{III.B.5. Isolated Propeller}

The Dymore and CAMRAD II propeller models are verified against each other. As mentioned in the Analytical Models section, the propeller blades in Dymore are modeled as flexible while in CAMRAD II they are modeled as rigid. Figure $11 \mathrm{~b}$ shows the pitch angle that resulted in zero torque at a particular velocity for an isolated propeller at two operating conditions: at an altitude of 8000 feet and a propeller speed of 2250 RPM; and at sea level for 2700 RPM. Overall good agreement is shown between CAMRAD II and Dymore predictions.

The thrust versus pitch angle for an isolated propeller is shown in Figure 11c for the two cases tested. At sea level conditions and 2700 RPM, Dymore and CAMRAD II agree if the propeller is not stalling, approximately between collective angles of +/- 10 degrees. At 8000 feet and 2250 RPM, CAMRAD II and Dymore again show good agreement in between the two stall points. The two analyses show some discrepancy beyond propeller stall, which is beyond the scope of current study.

Figure $11 \mathrm{~d}$ shows the power vs. pitch angle for the two cases. Similar conclusions are drawn as in the thrust predictions. 


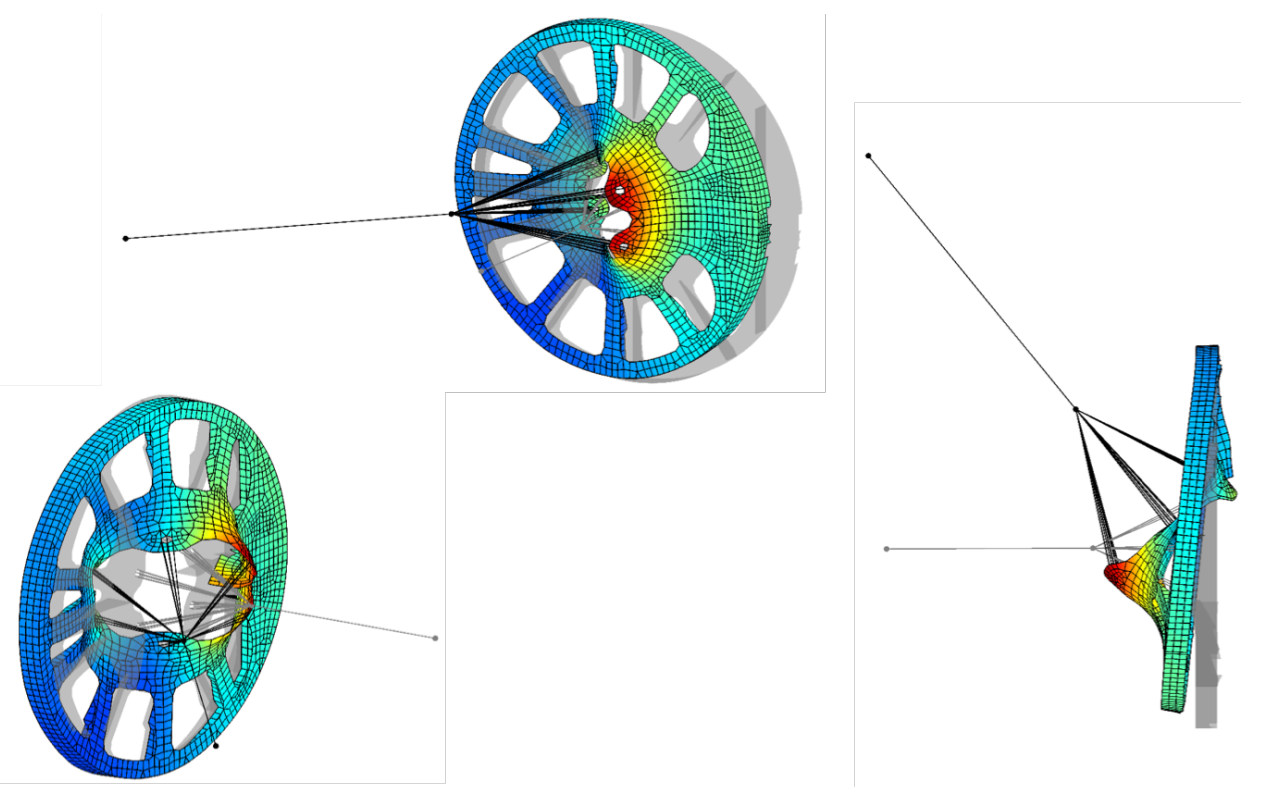

Figure 6: Version 2: Full FEM Shapes: Mode 5 Detail
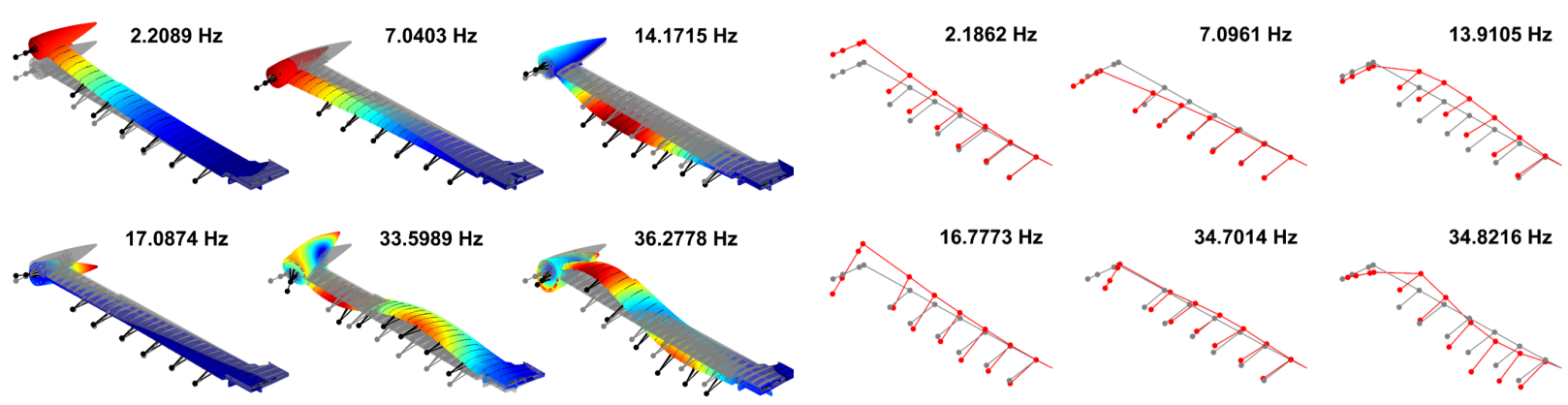

(a) Full FEM

(b) Beam model

Figure 7: Version 3: Full FEM and Equivalent Beam Model

\section{III.C. Whirl Flutter Stability}

The whirl flutter stability of three wing design versions is presented. The whirl flutter stability of the X-57 Maxwell aircraft is studied with the semi-span analytical models. CAMRAD II and Dymore use different methods to calculate the wing damping. CAMRAD calculates the wing damping directly through eigenanalysis of the system equations of motion. Dymore, instead, uses the Prony method to identify the wing damping based on the wing transient response. ${ }^{7}$ In addition, all the calculations of whirl flutter stability in CAMRAD II are carried out without wing aerodynamics because it uses a modal representation of the wing.

\section{III.C.1. Whirl Flutter Stability: Version 1}

The frequency and damping ratio of the wing bending and torsional modes are predicted for a range of airspeeds, as shown in Figure 12. The potential critical mode, that is the mode with the possible onset of whirl flutter, is identified as the torsional modes by both analyses. The flutter speed is found around 200 knots. The calculations, however, are conducted without including wing aerodynamics.

The whirl flutter stability of the X-57 Maxwell is recalculated using Dymore including a quasi-steady wing aerodynamics model. Figure 13 shows the wing frequency and damping variation with airspeed for the bending and torsional modes. The wing aerodynamics has a stabilizing effect on the torsional modes as the torsional flutter is seen to be eliminated when wing aerodynamics is included,Figure 13. The damping of the first wing bending mode is 


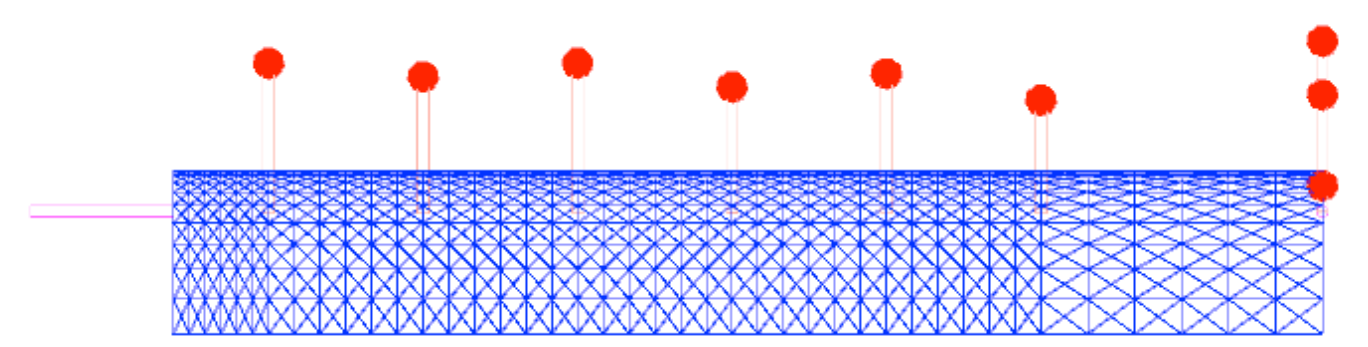

Figure 8: Semi-Span X-57 Maxwell Wing/Pylon Dymore Model

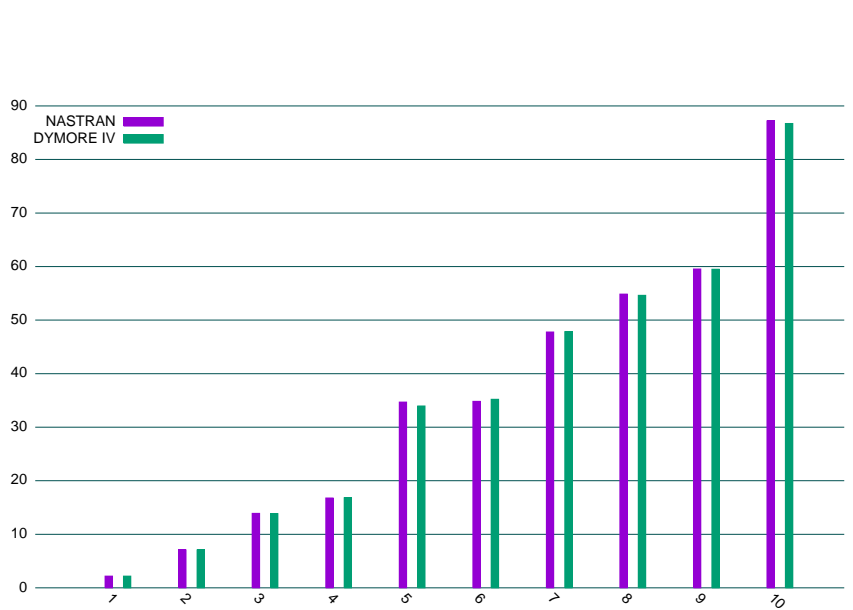

Figure 9: Frequencies of Wing/Pylon Model; Version 3

\begin{tabular}{rrrr}
\hline Mode & NASTRAN & Dymore & $\begin{array}{r}\mathrm{N} / \mathrm{D} \\
\mathrm{Hz}\end{array}$ \\
& $\mathrm{Hz}$ & (\% Error $)$ \\
\hline 1 & 2.18 & 2.19 & 0.5 \\
2 & 7.09 & 7.11 & 0.3 \\
3 & 13.91 & 13.87 & -0.3 \\
4 & 16.77 & 16.89 & 0.7 \\
5 & 34.70 & 34.11 & -1.7 \\
6 & 34.82 & 35.35 & 1.5 \\
7 & 47.78 & 47.89 & 0.2 \\
8 & 54.86 & 54.70 & -0.3 \\
9 & 59.55 & 59.56 & 0.0 \\
10 & 87.21 & 86.74 & -0.5 \\
\hline
\end{tabular}

Table 1: Frequencies of Wing/Pylon Model; Version 3

also shown to increase substantially when including the wing aerodynamics. In contrast, the wing aerodynamics has a negligible effect on the damping of the inplane (knife-edge) bending mode.

In addition, the wing aerodynamics has a small effect on the frequencies of the wing bending (out-of-plane and inplane) modes. However, the wing torsional frequency is affected by the wing aerodynamics noticeably, showing a different trend or variation with airspeed for with and without wing aerodynamics.

\section{III.C.2. Whirl Flutter Stability: Version 2}

Due to the equivalent beam not being able to properly represent the flexible faceplate, only a CAMRAD II analysis was performed. The CAMRAD II frequencies and damping ratios are shown in Figure 14. The flexibility of the faceplate caused large rotations in both mode 3 and 4 in the pitch direction, and the yaw flexibility was introduced through mode 5.

\section{III.C.3. Whirl Flutter Stability: Version 3}

Figures 15 shows the Dymore and CAMRAD II frequencies and damping of a semi-span X-57 Maxwell model in cruise, at a density altitude of 8000 feet and a propeller speed of 2250 RPM. The frequencies of the first (first bending), second (knife-edge), and fourth (torsion) modes show good agreement with CAMRAD II predicting a slightly lower frequency for the fourth mode at higher free stream velocities. The damping ratios agree well for the second and fourth modes with Dymore predicting a slight lower damping ratio for the second mode at higher free stream velocities. While the first predicted mode (first bending) agrees between Dymore and CAMRAD II, Dymore predicted a lower damping ratio as velocity increases.

Figure 16 shows the Dymore frequency and damping of a semi-span X-57 Maxwell model in cruise conditions with no wing aerodynamics, with quasi-steady wing aerodynamics, and with unsteady wing aerodynamics. While the frequencies of the lower two modes are similar among the three cases, the fourth mode (torsional) frequency increases drastically from the case with no wing aerodynamics to the cases that include wing aerodynamics as the velocity 
increases. The damping of the first mode is also very different between the case of no wing aerodynamics and those including wing aerodynamics, with the wing aerodynamics increasing the damping ratio of the mode. The damping on the fourth mode is the only mode where the quasi-steady and unsteady aerodynamics differ significantly, with the unsteady aerodynamics contributing to a higher damping ratio.

Figure 17 shows the Dymore frequency and damping of a semi-span X-57 Maxwell model at sea level conditions and at 2700 RPM with no wing aerodynamics, with quasi-steady wing aerodynamics, and with unsteady wing aerodynamics. The modes in Figure 16 at high altitude present an overall higher damping than those at sea level, Figure 17. The damping of the fourth mode has a similar trend as those at higher altitude conditions (Figure 16) for quasi-steady and unsteady wing aerodynamics.

\section{Summary and Conclusions}

Two multibody dynamics codes, Dymore and CAMRAD II, are used to study the whirl flutter stability of the experimental NASA aircraft designated the X-57 Maxwell. Good agreements are shown for the predictions of propeller thrust and power between CAMRAD II and Dymore. The natural frequencies and mode shapes of the semi-span model are compared between Dymore and NASTRAN predictions and show good correlations. For versions two and three, the first four modes of the semi-span model, which are first bending, inplane (knife-edge) bending, second bending, and torsion, are examined for whirl flutter stability. The loads and power of an isolated propeller are predicted by the multibody dynamics codes and compared to each other. The thrust and power of the propeller agreed well within the apparent stall angles and diverged from each other afterwards. The whirl flutter analysis from both CAMRAD II and Dymore showed similar frequencies and damping ratios for each mode when no wing aerodynamics were used although Dymore predicted slightly lower damping ratios than CAMRAD II. CAMRAD II results captured a whirl flutter case for the wing design version 2, which prompted a redesign of the pylon mount system to eliminate the issue. When quasi-steady and unsteady aerodynamics were included in the Dymore analysis, the damping of the fundamental bending mode increased dramatically with velocity, and became the most stable among the four modes. This also corresponds to a decrease in the damped frequency compared to the no wing aerodynamics case, as expected. The inplane bending mode was not affected significantly by including wing aerodynamics. The torsion frequencies increased with velocities when wing aerodynamics were applied. The torsion damping ratio varied depending on the type of aerodynamics, showing higher damping ratio with unsteady aerodynamics than quasi-steady aerodynamics. As altitude increases, density decreases, and the damping ratios decrease. Overall the damping ratios of all the semispan model modes are positive for the latest wing version, indicating that the design is clear of whirl flutter for the symmetric modes up to 500 knots, well above the operating range of the X-57.

\section{Acknowledgments}

The first two authors were funded by NASA Langley through National Institute of Aerospace. The authors would like to thank Jeffrey Viken and Nicholas Borer (NASA Langley Research Center), and Olivier Bauchau (University of Maryland, College Park) for their support for this study.

\section{References}

${ }^{1}$ Borer, N. K., Patterson, M. D., Viken, J. K., Moore, M. D., Bevirt, J., Stoll, A. M., and Gibson, A. R., "Design and Performance of the NASA SCEPTOR Distributed Electric Propulsion Flight Demonstrator," 16th AIAA Aviation Technology, Integration, and Operations Conference.

${ }^{2}$ Taylor, E. S. and Browne, K. A., "Vibration Isolation of Aircraft Power Plants," Aero Sci 6, no. 2, 43, 1938.

${ }^{3}$ Abbott, F. T., Kelly, H., and Hampton, K. D., "Investigation of Propeller-Power-Plant Autoprecession Boundaries for a Dynamic-Aeroelastic Model of a Four-Engine Transport Airplane," NASA tech. Note D-1806, 1963.

${ }^{4}$ Houbolt, J. C. and Reed III, W. H., "Propeller-Nacelle Whirl Flutter," IAS 29th Annual Metting, January 23-25 1961.

${ }^{5}$ Reed III, W. H., "Propeller-Rotor Whirl Flutter: A State-of-the-Art Review," Sound and Vibration, Vol. 4, November 1966, pp. 526-544.

${ }^{6}$ Johnson, W., "Technology Drivers in the Development of CAMRAD II," American Helicopter Society Aeromechanics Specialists Conference, San Francisco, CA, January 19-21 1994.

${ }^{7}$ Bauchau, O. A., DYMORE USERS MANUAL, 2016.

${ }^{8}$ Shen, J. and Kang, H., "Comparison Study of Tiltrotor Whirl Flutter Using Two Rotorcraft Comprehensive Analyses," AHS International Technical Meeting on Aeromechanics Design for Vertical Lift, San Francisco, CA, January 2016.

${ }^{9}$ Kang, H., Shen, J., and Kreshock, A. R., "Parametric Study of Tiltrotor Whirl Flutter Using Two Rotorcraft Comprehensive Analyses," American Helicopter Society 72nd Annual Forum Proceedings, West Palm Beach, FL, May 2016.

${ }^{10}$ Kreshock, A. R., Thornburgh, R., Yeo, H., and Kang, H., "Validation of Comprehensive Modeling of the Wing and Rotor Aeroelastic Test System," American Helicopter Society 72nd Annual Forum Proceedings, West Palm Beach, FL, May 2016. 
${ }^{11}$ Kreshock, A. R. and Yeo, H., "Tiltrotor Whirl-Flutter Stability Predictions Using Comprehensive Analysis," AIAA Technical Conference, 2017.

${ }^{12}$ Floros, M., Shen, J., Lee, M. K., , and Hwang, S., "Loads and Stability Analysis of an Unmanned Tilt Rotor," American Helicopter Society 62nd Annual Forum Proceedings, Phoenix, AZ, May 2006, p. 19.

${ }^{13}$ Hoover, C., Shen, J., Kang, H., and Kreshock, A. R., "Pylon Conversion Proprotor Loads and Whirl Flutter Stability of a Tiltrotor Wind Tunnel Model," American Helicopter Society 73rd Annual Forum Proceedings, Fort Worth, Texas, May 9-11 2017.

${ }^{14}$ Bauchau, O., Bottasso, C., and Nikishkov, Y., "Modeling Rotorcraft Dynamics with Finite Element Multibody Procedures," Mathematical and Computer Modeling, Vol. 33, 2001, pp. 1113-1137.

${ }^{15}$ Peters, D. and He, C., "Finite State Induced Flow Models. Part I: Two Dimensional Thin Airfoil," Aircraft, Vol. 32(2), 1995, pp. $313-322$.

${ }^{16}$ NASTRAN Quick Reference Guide, The MacNeal Schwendler Corporation. 


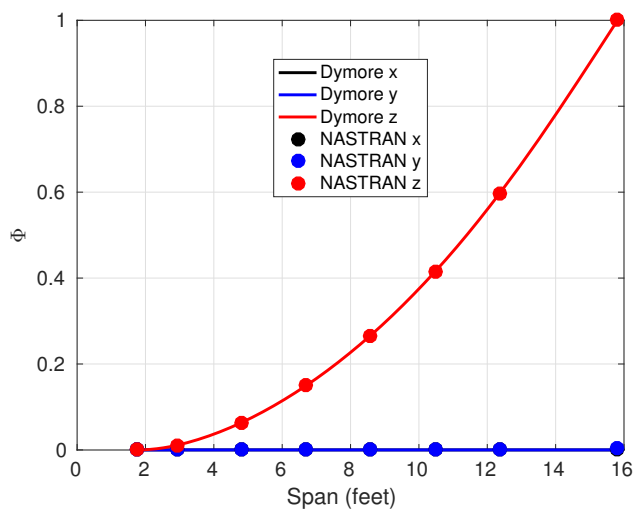

(a) Mode 1 (First Bending); Displacements

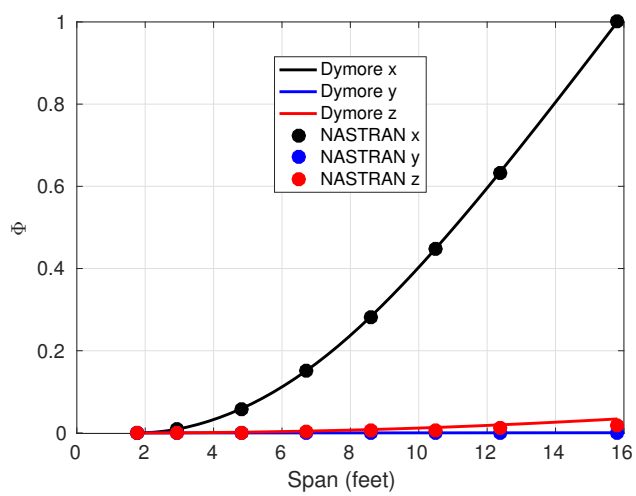

(c) Mode 2 (First In-Plane); Displacements

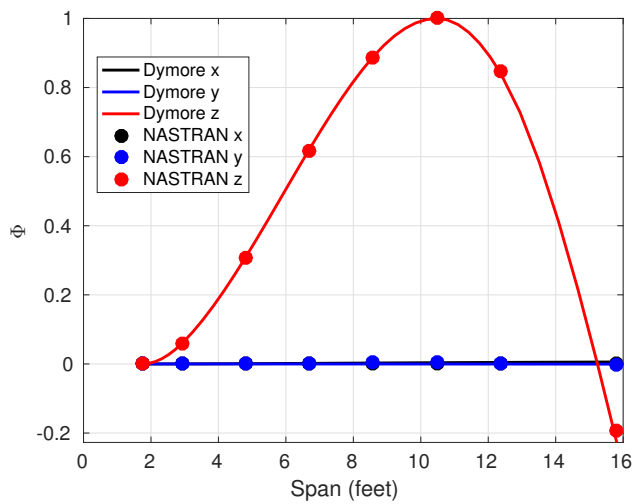

(e) Mode 3 (Second Out-of-Plane); Displacements

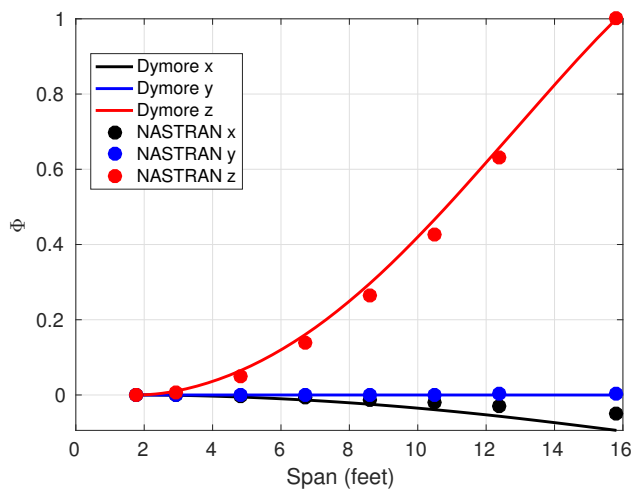

(g) Mode 4 (First Torsion); Displacements

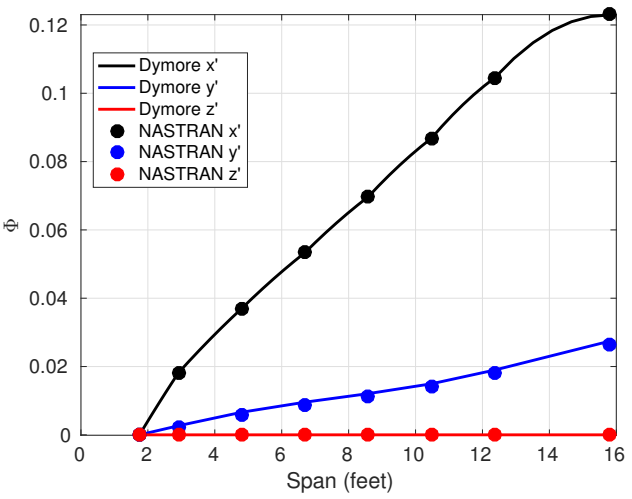

(b) Mode 1 (First Bending); Rotations

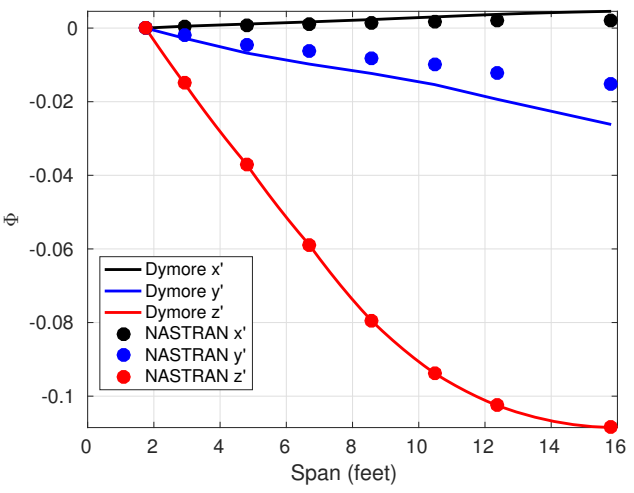

(d) Mode 2 (First In-Plane); Rotations

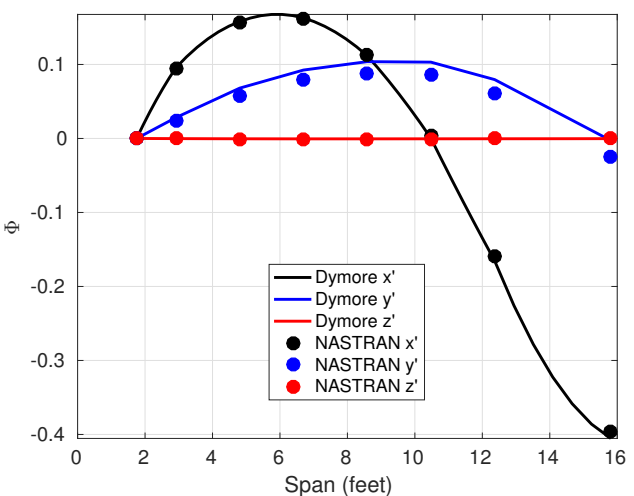

(f) Mode 3 (Second Out-of-Plane); Rotations

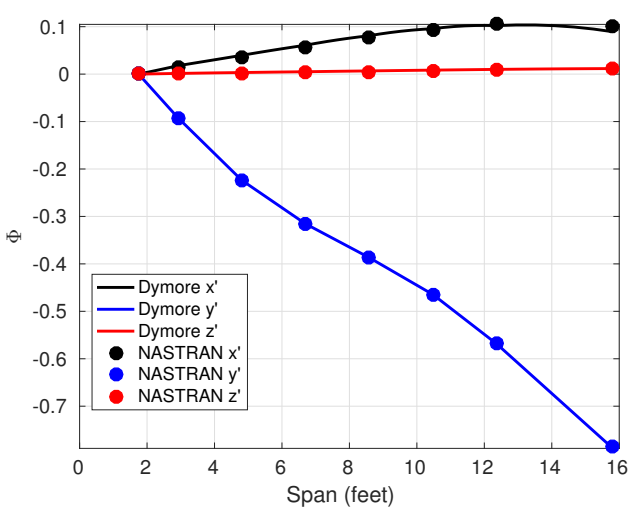

(h) Mode 4 (First Torsion); Rotations

Figure 10: Comprison of NASTRAN and Dymore Predictions of Wing Modes; Version 3 


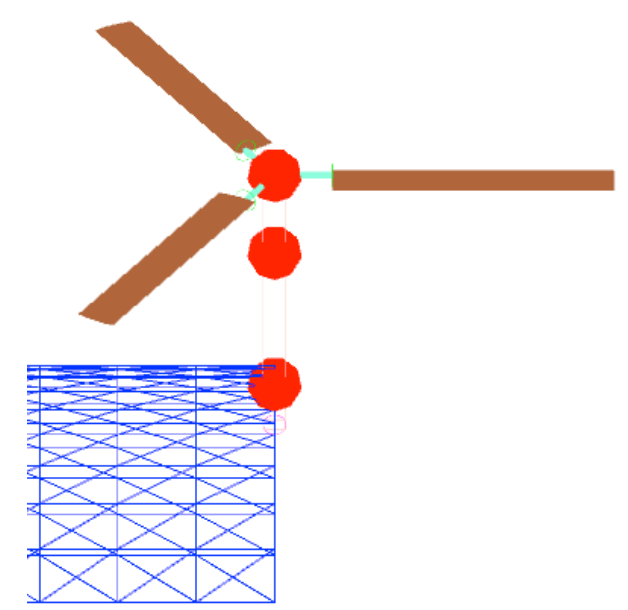

(a) X-57 Maxwell Propeller Dymore Model

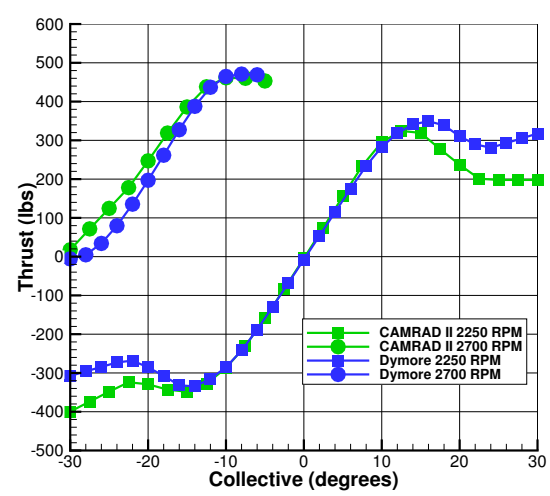

(c) Propeller Thrust

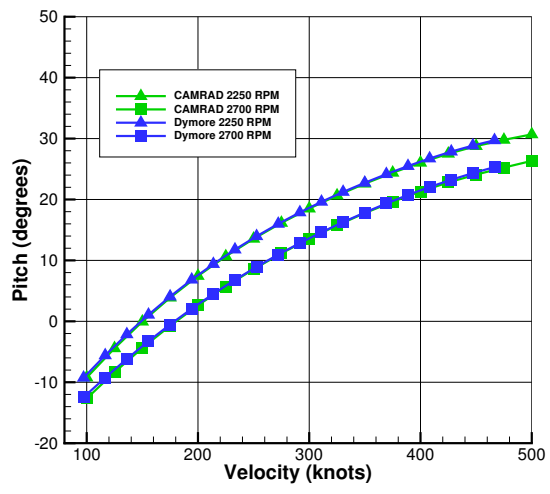

(b) Propeller Pitch Change Under Windmilling

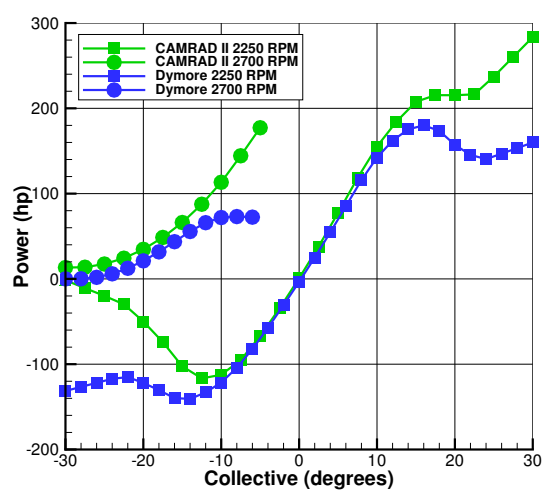

(d) Propeller Power

Figure 11: Isolated Propeller Performance

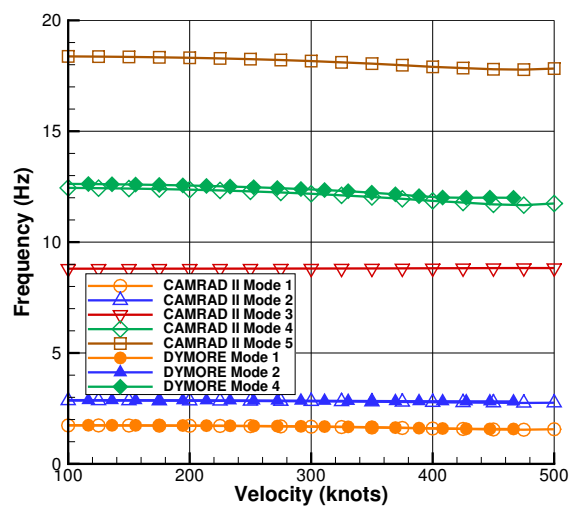

(a) Frequency

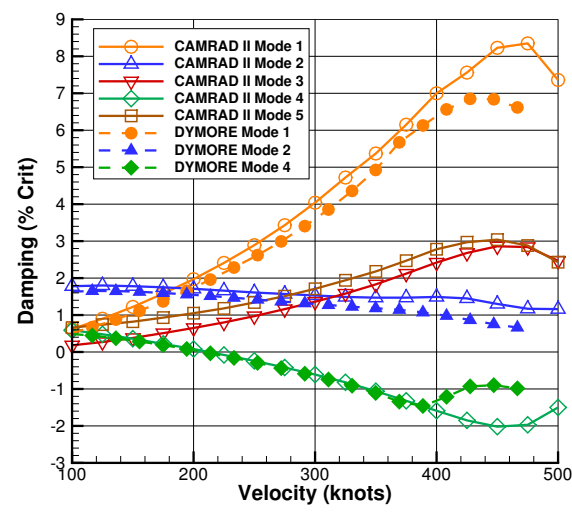

(b) Damping

Figure 12: Frequency and Damping of Semi-Span X-57 Maxwell Model; Without Wing Aerodynamics; RPM 2250; Version 1 


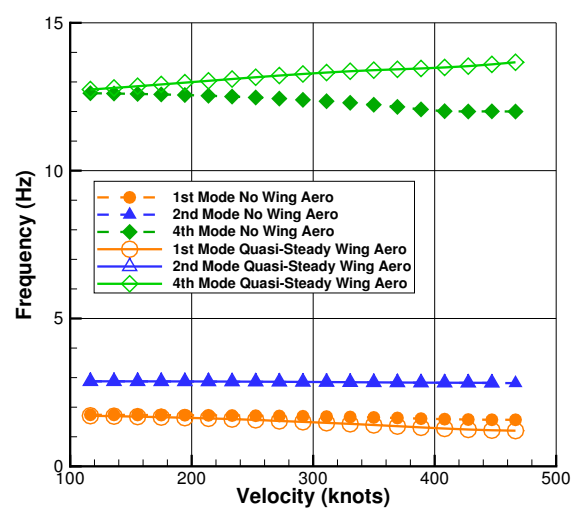

(a) Frequency

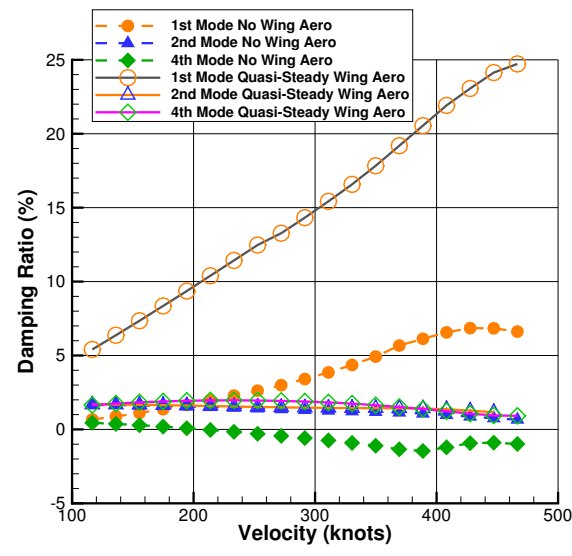

(b) Damping

Figure 13: Dymore Frequency and Damping of Semi-Span X-57 Maxwell Model; With Wing Aerodynamics; Version 1

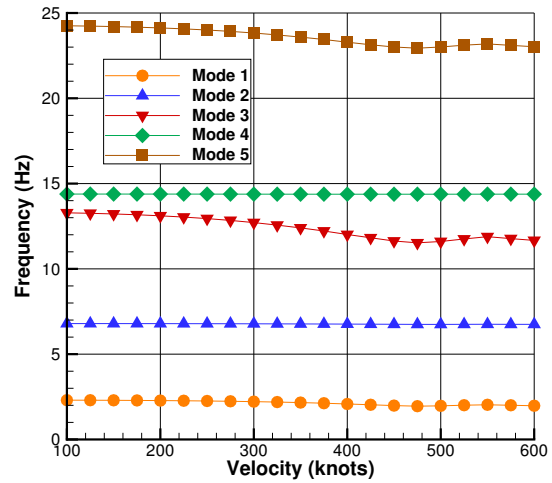

(a) Frequency

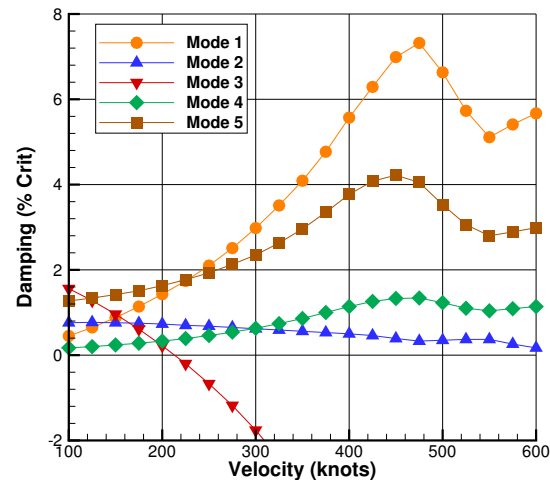

(b) Damping

Figure 14: CAMRAD II Frequency and Damping of Semi-span X-57 Maxwell Model; Without Wing Aerodynamics; 2250 RPM; Version 2

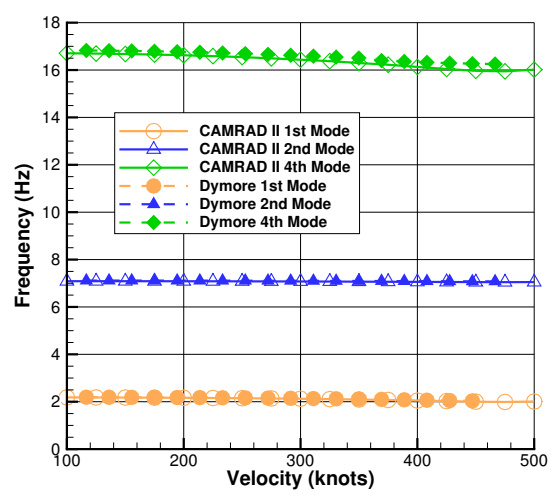

(a) Frequency

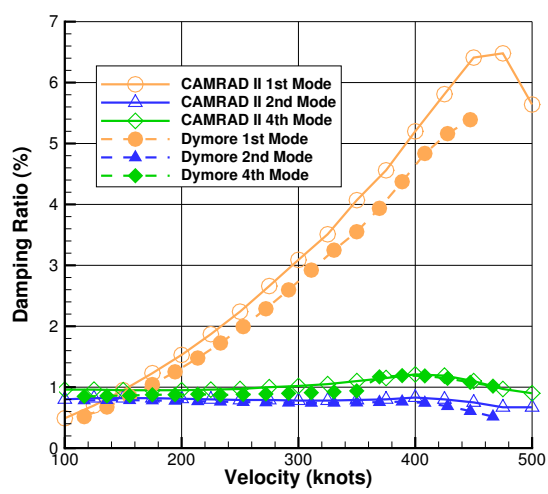

(b) Damping Ratio

Figure 15: Frequency and Damping of Semi-Span X-57 Maxwell Model; Without Wing Aerodynamics; RPM 2250; Version 3 


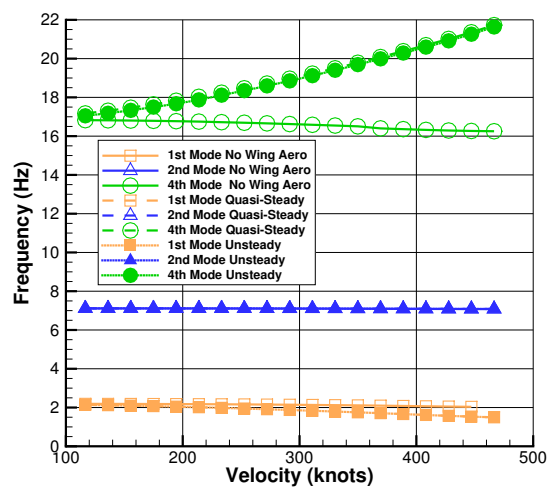

(a) Frequency

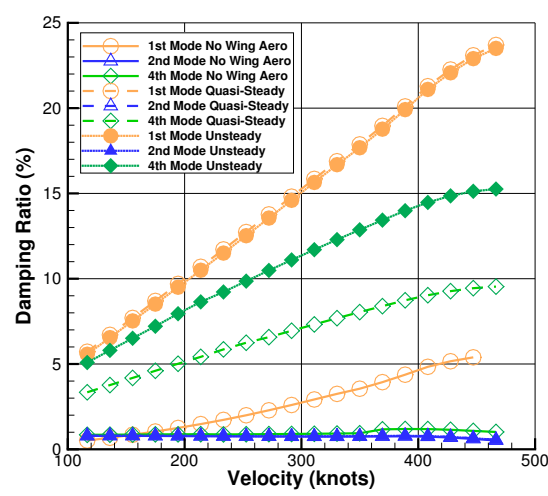

(b) Damping Ratio

Figure 16: Dymore Frequency and Damping of Semi-Span X-57 Maxwell Model; Comparison of Adding Wing Aerodynamics; RPM 2250; Version 3

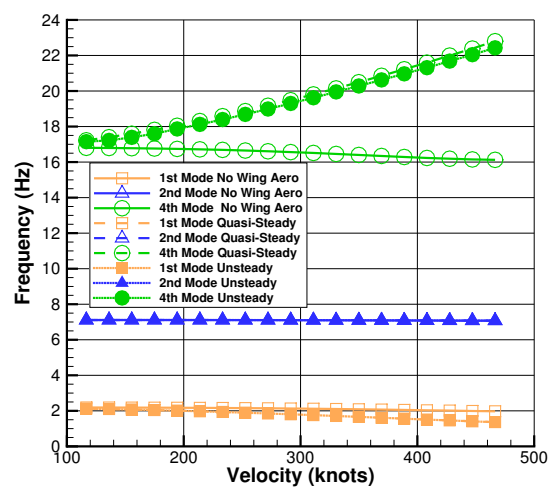

(a) Frequency

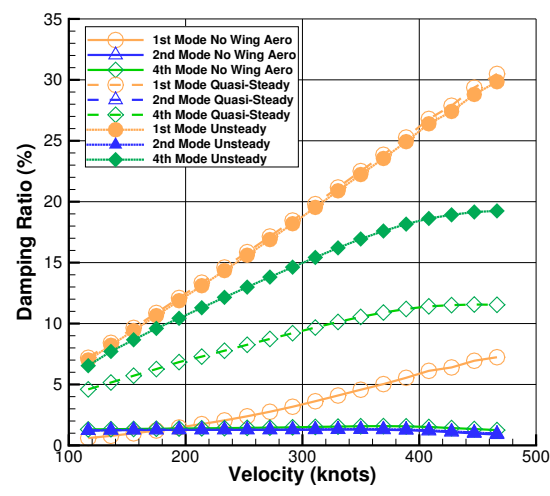

(b) Damping Ratio

Figure 17: Dymore Frequency and Damping of Semi-Span X-57 Maxwell Model; Comparison of Adding Wing Aerodynamics; RPM 2700; Version 3 